\title{
Intelligent ubiquitous IT policy and its industrial services
}

\author{
Hang Bae Chang · Jianhua Ma - Seng W. Loke • \\ Hans-Dieter Zimmermann - Zhuowei Li
}

Received: 22 August 2011 / Accepted: 26 August 2011 / Published online: 28 October 2011

(C) Springer Science+Business Media, LLC 2011

\begin{abstract}
The core bases of intelligent ubiquitous computing environments are ubiquitous network infrastructure and the related services. Telecommunication service industry includes such ubiquitous network infrastructure and most of related services, and it leads to simultaneous growth of network, terminal, software and contents etc., by placing at the top of value chain of IT industry. In this special issue, we have discussed current IT-related issues, policy trends and new industrial services which will lead to successful transfer toward intelligent ubiquitous society which is a new paradigm of future.
\end{abstract}

\author{
H. B. Chang $(\varangle)$ \\ Department of Business Administration, \\ Daejin University, Pocheon, Korea \\ e-mail: hangbae.chang@gmail.com \\ J. Ma \\ Faculty of Computer \& Information Sciences, \\ Hosei University, Tokyo, Japan \\ e-mail: jianhua@hosei.ac.jp \\ URL: http://cis.k.hosei.ac.jp/ jianhua/

\section{S. W. Loke} \\ Department of Computer Science and Computer Engineering, \\ La Trobe University, Bundoora, Australia \\ e-mail: s.loke@latrobe.edu.au \\ URL: http://homepage.cs.latrobe.edu.au/sloke/
}

\section{H.-D. Zimmermann}

Swiss Institute for Information Research SII,

University of Applied Sciences, St. Gallen, Switzerland

e-mail: hans-dieter.zimmermann@fh-htwchur.ch

URL: http://www.hdzimmermann.net/

\section{Z. Li}

Windows Azure, Microsoft Corporation,

Seattle, WA, USA

e-mail: Zhuowei.Li@microsoft.com
Keywords Intelligent ubiquitous - IT policy · Industrial service

\section{Introduction}

Due to development of IT technology, various types of computer became the parts of human beings, objects, and environment, and intelligent ubiquitous computing environment, which is connected with one another to assist human life, is rapidly being created. As the ubiquitous environment is gaining more attention as an open platform for integrating separate distributed services, businesses and individuals are looking into the possibility of providing their services in such an environment (Prince and Adam 2005). To this end, diversified services in a ubiquitous environment are being actively experimented in extensive fields. Ubiquitous service can be defined in various ways. It is defined as Radio Frequency Identification (RFID) applicable service, Home Network Service, Telematics Service and Location Information Service, which are the center of Ubiquitous Sensor Network (USN), and related wire/wireless telecommunication and broad casting service etc. are included in broad sense (Mattern 2004).

In this special issue, we have discussed current IT-related issues, policy trends and new industrial services which will lead to successful transfer toward intelligent ubiquitous society which is a new paradigm of future. More specifically, re-establishment of national vision, modification of regulation system, introduction to new industrial services and activation, expansion of IT industry infrastructure, adverse effects of intelligent ubiquitous systems, the role of individuals as consumers, employees, and citizens, etc. have been discussed. 


\section{Papers in this issue}

This issue presents recent progress on intelligent ubiquitous IT policy, its industrial services and research strategies, methodologies together with particular applications of ubiquitous technology to real life problem. The first paper have present a novel framework for intelligent model design of cluster supply chain, and the second paper have researched the common criteria methodology for secure ubiquitous environment construction and emphasized importance of private information security in ubiquitous environment. The third paper has analyzed effects of information security investments to cope with the changing corporate business environment, and the fourth paper propose a virtual node based ring-like storage system architecture and data placement scheme for delay-sensitive ubiquitous applications. The last paper have investigated current situation of Taiwan MOE ICT security development and provide a case study.

(1) The paper 'Intelligent Model Design of Cluster Supply Chain with Horizontal Cooperation' by Jizi Li, Naixue Xiong, Chunling Liu, and Jong Hyuk Park have presented a novel framework for intelligent model design of cluster supply chain in which there are a multiple of rivals or potential competitors in the proximity for each member along supply chain. They have provided a novel framework and approach to design cluster supply chain without across-chain horizontal cooperation, then by introducing item allocation proportion of vertical and horizontal cooperation $(\alpha: 1-\alpha)$, the cluster supply chain design with cross-chain horizontal cooperation was developed, then presents a hybrid method to find solution, at last, computational study has been presented to investigate values of decision variables and their influence on cluster supply chain design.

(2) The paper 'Study on the Common Criteria Methodology for Secure Ubiquitous Environment Construction' by Soo-Young Kang emphasized the importance of private information security is rising as the ubiquitous environment settles down with the methods to secure private data. According to this study, when information is exposed, in terms of U-Healthcare or U-Home network that deals with personal data, both the privacy and life could be threatened. However, when the verification system based on the ubiquitous is not prepared, private information can be exposed and cause system failure or errors. Therefore, secured assurance and verification should be proceeded in the established ubiquitous environment with a common evaluation standard. Presently, the IC card and smart cards are increased with evaluation and certification through the $\mathrm{CC}$, but the core technology of the ubiquitous environment which is RFID and the sensors are not still unprepared. Hence, both the identification system and ubiquitous environments should be evaluated according to the variety of base systems, and further studies must be conducted to develop a safe ubiquitous environment.

(3) The paper 'An Analysis on Effects of Information Security Investments: A BSC Perspective' by Hee-Kyung Kong, Tae-Sung Kim, and Jungduk Kim sought to present BSC as an analytic tool for enterprise-wide strategies of information security in response to the changing corporate business environment. The BSC perspective of investment strategies of information security were set up considering the characteristics of information security investment and the validity of the BSC perspective investment strategies for information security were tested by finding a causal link between the investment strategies and performance. According to the study results, the asset risk management and work process control above all should be strengthened through the building of a technological/human infrastructure from a learning and growth perspective.

(4) The paper 'An efficient and scalable ubiquitous storage service for delay-sensitive IT applications' by Feilong Tang, Song Guo, Minyi Guo, and Qiang Wang propose a virtual node based ring-like storage system architecture and data placement scheme for delay-sensitive ubiquitous applications. For improving the fault-tolerant ability of the storage service, we design a data replication approach based on a quorum-fault-tolerant state machine protocol. Moreover, we investigate a three-layered messaging protocol based on the heart beat mechanism to detect the node failure and maintain the ring topology. The theoretic analysis and experiment result demonstrate the feasibility and high operation efficiency of our ubiquitous storage service.

(5) The paper 'Establish a Secure and Trustworthy Ubiquitous ICT Environment for Educational Systems: A Case Study' by Yu-Hsiu Chuang, Chi-Yuan Chen, and TzongChen Wu investigated current situation of Taiwan MOE ICT security development and provide a case study. We discuss these challenges that educational systems would meet. Finally, we review their proposed solutions with policy management aspect, environment and technical aspect, and education aspect for improving ICT security environment in educational systems. It provides reference materials for establishing a secure and trustworthy ICT environment within educational systems.

(6) The paper 'Integrated inventory and transporta-tion decision for ubiquitous supply chain management' by Ki-sung Hong, Sang Soo Yeo, Hyoung Joong Kim, Ek Peng Chew, and Chulung Lee have developed a model to jointly consider the inventory and transportation decision for a supply chain system, and the main model deals with the quantity-based dispatch policy. As a result of 
this study, the quantity-based dispatch policy with pricing (QWP) significantly improve the total profit.

\section{References}

Mattern, F. (2004). Wireless future: Ubiquitous computing. In Proceedings of Wireless Congress 2004, Munich, Germany, November.
Prince, B. N. B. A., \& Adam, K. (2005). Keeping ubiquitous computing to yourself: A practical model for user control of privacy. International Journal of Human-Computer Studies, 63, 228-253. 